\title{
Achievable QoS in an Interference/Resource-Limited Shared Wireless Channel
}

\author{
Jeffrey M. Capone \\ Department of Electrical Engineering \\ Arizona State University, Tempe, AZ 85287-7206 \\ jcaponelasu . edu \\ Ioannis Stavrakakis \\ Department of Electrical and Computer Engineering \\ Northeastern University, Boston, MA 02115 \\ ioannis@cdsp.neu. edu
}

\begin{abstract}
In this work, the region of achievable QoS - which is central to the development of a call admission control mechanism - is precisely described for a system of heterogeneous VBR sources with real-time service constraints. The QoS for each application is defined in terms of a packet dropping probability. Packets may be dropped due to delay violations and channel induced errors. The shared transmission resources are defined to be the slots (packet transmission times) of a TDMA frame. The region of achievable QoS is precisely described for a interference/resource-limited network by considering the underlying TDMA-MAC structure and the physical channel. A simple DLC protocol that combats the effects of the wireless channel while satisfying the real-time requirements is proposed and its impact on the region of achievable QoS is evaluated. The results presented here clearly illustrate the negative impact of a poor channel and the positive impact of the employed DLC protocol on the achievable QoS.
\end{abstract}

Keywords

Quality of Service (QoS), TDMA, Scheduling, DLC.

\section{INTRODUCTION}

In integrated services wireless networks transmission resources are shared among geographically dispersed applications with diverse traffic characteristics and quality of service (QoS) requirements. In this work, the shared transmission resources are defined to be the slots (packet transmission times) 
of a TDMA frame. This resource structure has been widely considered in both cellular personal communication networks (PCN) [1] and wireless LANs, as well as in recent work toward the development of wireless ATM networks [2]. The QoS requirements for each application are defined in terms of a maximum tolerable packet delay and dropping probability. To provide QoS guarantees and use the bandwidth efficiently, call admission and scheduling functions are necessary.

A variety of scheduling policies have been proposed to support real-time traffic in a shared wireless environment $[3,4,5,6,7]$. In [3, 4], the performance of the scheduling policies are evaluated for different combinations of variable bit rate (VBR) sources, while in $[5,6,7]$, the scheduling policies are designed to meet (if achievable) the QoS required by the set of heterogeneous VBR applications. In [5], the authors determine a very conservative scheme and provide only sufficient conditions to ensure that the scheduling policy can deliver service satisfying the delay constraints of the VBR sources and meet their deterministic QoS guarantees. Probabilistic QoS guarantees are considered in $[6,7]$ and in this paper. For example, packets from a particular source (such as voice or video) might only need to meet their delay constraint $99 \%$ of the time, and can tolerate being dropped otherwise. In this work, necessary and sufficient conditions in order for the probabilistic QoS to be achieved are presented, leading to bandwidth efficient call admission control and scheduling algorithms.

In a wireless network, achievable QoS is shaped not only by the amount of available resources and the employed transmission scheduling, but also by the channel quality (interference). As a consequence, the region of achievable QoS vectors is shaped by the packet discarding process at both the transmitter and the receiver due to resource and interference limitations, respectively. In addition, the performance - and therefore the region of achievable QoS can be enhanced by considering a properly designed DLC protocol for this environment. Such a protocol is also considered in this paper.

\section{DESCRIPTION OF THE SYSTEM MODEL}

Consider a system where $N$ heterogeneous VBR sources compete for $T$ slots of an up-link TDMA channel. At the beginning of each frame $n$ each source $i$ requests a random number of slots denoted by $\lambda_{i}(n)$. If the aggregate demand in frame $n, \sum_{i=1}^{N} \lambda_{i}(n)$, exceeds the number of slots available to the VBR traffic - referred to as an overloaded frame - then decisions must be made regarding the amount of service that will be provided to each source. The number of slots, $a_{i}^{f}(n)$, under scheduling policy $f$ allocated to source $i$ may be less than what is required by that source, $\lambda_{i}(n)$, due to resource limitation. Packets from a source which do not receive service over a frame following their arrival are considered to have excess delay and are dropped at the source. 
The effects of the wireless channel are modeled as in [8], where a Gaussian noise channel with random bit erasure interference is considered. The erasure process might be produced by a burst noise process which produces bursts of erasures. However, in [8], an interleaver/deinterleaver is employed to turn the erasure bursts into statistically independent bit erasures. Therefore, in this system, packet erasures are considered to be statically independent and occur when the interference in the channel is such that the packet is corrupted in manner that it can not be corrected. The corrupted transmitted packets are discarded (dropped) at the receiver.

The event that the packet is corrupted and therefore dropped is a function of interference in the channel, the transmitted power, the coding scheme, and the packet length. Let $Z$ be an indicator function of a packet erasure. That is,

$Z=\left\{\begin{array}{ll}1 & \text { if the packet is corrupted } \\ 0 & \text { otherwise }\end{array}\right.$.

Therefore, the expected value of $Z, E[Z]=\beta$, is the probability that a packet is corrupted by the channel and dropped.

Considering the combined impact of scheduling policy $f$ and the physical channel, the number of packets from source $i$ dropped in frame $n$ due to the above competition for the resources and the interference in the channel is given by,

$$
d_{i}^{f}(n)=\left\{\begin{array}{ll}
\sum_{m=1}^{\lambda_{i}(n)} Z_{m} & \text { if } \sum_{j=1}^{N} \lambda_{j}(n) \leq T \\
\lambda_{i}(n)-a_{i}^{f}(n)+\sum_{m=1}^{a_{i}^{f}(n)} Z_{m} & \text { if } \sum_{j=1}^{N} \lambda_{j}(n)>T
\end{array} .\right.
$$

$Z_{m}$ is an indicator function associated with the transmission of the $m^{\text {th }}$ packet from source $i$. Let $d_{i}^{f}=E\left[d_{i}^{f}(n)\right], a_{i}^{f}=E\left[a_{i}^{f}(n)\right]$ and $\lambda_{i}=E\left[\lambda_{i}(n)\right]$ be the (assumed time invariant) expected values of the associated quantities.

Suppose that the QoS requirement of application $i$ is defined in terms of a maximum tolerable average per frame packet dropping rate $d_{i}, 1 \leq i \leq N$. Then the QoS vector associated with these applications can be defined in terms of the (performance) packet dropping rate vector $\mathbf{d}=\left(d_{1}, d_{2}, \ldots, d_{N}\right)$. When the QoS requirement of the application $i$ is defined in terms of a maximum tolerable packet dropping probability $p_{i}$, the corresponding packet dropping rate $d_{i}$ is easily determined by $d_{i}=\lambda_{i} p_{i}$.

The first question addressed in Section 3.1 is whether (under the given channel conditions) a given QoS vector $\mathbf{d}$ is achievable under some policy $f$. The results from Section 3.1 are modified to reflect the impact of the proposed real-time DLC protocol and are presented in Section 3.2. The second question, addressed in Section 4, is concerned with the design of scheduling policies that deliver an achievable target $\mathrm{QoS}$ vector $\mathbf{d}$. 


\section{DETERMINATION OF THE REGION OF ACHIEVABLE QOS VECTORS}

The establishment of the region of achievable QoS vectors is based on a set of inequalities and an equality constraint derived by employing work-conserving arguments. The superscript $f$ is used to denote the employed packet scheduling policy. It is assumed that the scheduling polices are work-conserving (that is, non-idling) and induce a performance vector $\mathrm{d}^{f}$.

\subsection{Achievable QoS Provided by the Underlying MAC and Physical Channel}

Let $S=\{1,2, \ldots, N\}$ be the set of all sources and $d_{S}^{f}$ denote the average system packet dropping rate under scheduling policy $f$, denoted by,

$$
d_{S}^{f} \triangleq E\left[\sum_{i=1}^{N} d_{i}^{f}(n)\right]=\sum_{i=1}^{N} E\left[d_{i}^{f}(n)\right]=\sum_{i=1}^{N} d_{i}^{f} .
$$

Let $\lambda_{g}(n)$ denote the aggregate arrival rate from sources in set $g, g \subseteq S$. That is, $\lambda_{g}(n)=\sum_{i \in g} \lambda_{i}(n)$.

Summing (2) over all sources $i \in S$ and by considering the expected value of the associated quantity, the average system packet dropping rate under work-conserving scheduling policy $f$ is derived and it is given by,

$$
\begin{array}{r}
d_{S}^{f}=\left\{E\left[\lambda_{S}(n) \mid \lambda_{S}(n)>T\right]-T(1-\beta)\right\} P\left(\lambda_{S}(n)>T\right) \\
+\beta\left\{E\left[\lambda_{S}(n) \mid \lambda_{S}(n) \leq T\right]\right\} P\left(\lambda_{S}(n) \leq T\right) .
\end{array}
$$

As it can be seen from (4), $d_{S}^{f}$ is independent from the policy $f$; it only depends on the aggregate arrival process, the number of resources $T$, and the channel characteristics $\beta$. Therefore, the system dropping rate, $d_{S}^{f}$, is conserved under any work-conserving policies $f$ and is denoted as $b_{S}$.

Let $d_{g}^{f}$ denote the average subsystem $g$ packet dropping rate under policy $f$, defined by, $d_{g}^{f} \triangleq E\left[\sum_{i \in g} d_{i}^{f}(n)\right]=\sum_{i \in g} E\left[d_{i}^{f}(n)\right]=\sum_{i \in g} d_{i}^{f}, \quad g \subset S$. That is, $d_{g}^{f}$ is equal to the aggregate packet dropping rate associated with sources in group $g$ only, under policy $f$; all $N$ sources in $S$ are assumed to be present and served under policy $f$.

Let $b_{g}$ denote the lower bound on the aggregate packet dropping rate for sources in $g$. This bound is equal to the packet dropping rate of a system in which only sources in $g$ are present and served under a work-conserving 
policy; sources in set $\{S-g\}$ are considered to be removed. It is given by,

$$
\begin{array}{r}
b_{g}=\left\{E\left[\lambda_{g}(n) \mid \lambda_{g}(n)>T\right]-T(1-\beta)\right\} P\left(\lambda_{g}(n)>T\right) \\
+\beta\left\{E\left[\lambda_{g}(n) \mid \lambda_{g}(n) \leq T\right]\right\} P\left(\lambda_{g}(n) \leq T\right) .
\end{array}
$$

It is apparent that no policy can deliver a lower dropping rate than $b_{g}$ to sources in set $g$, when all sources in $S$ are present. It can be seen that this lower bound is attained by all policies $f$ which give service priority to packets from sources in set $g$ over those in the complement set $\{S-g\}$. It has been shown in [6] that in an error-free channel, the following conditions,

$$
\begin{aligned}
d_{g} & \geq b_{g} \\
d_{S} & =b_{S}
\end{aligned} \quad \forall g \subseteq S
$$

are necessary and sufficient in order for a QoS vector $\mathbf{d}=\left(d_{1}, d_{2}, \ldots, d_{N}\right)$ to be achieved by some scheduling policy $f$. This result can be extended to account for the channel quality provided that $b_{g}$, given in (5), is a super-modular set function; a detailed proof can be found in [9].

Let $\mathcal{D}$ denote the collection of all vectors $d$ satisfying (6) and (7). Then by definition $\mathcal{D}$ is a convex polytope. Using results from convex polytopes, any vector in the set $\mathcal{D}$ can be expressed as a convex combination of extreme points (vertices) of $\mathcal{D}$; that is, $\mathcal{D}$ may be expressed as the convex hull of its extreme points, $\mathcal{D}=\operatorname{conv}[\exp (\mathcal{D})]$.

In addition, from the polytope structure and the super-modularity property of the set function $b_{g}$, it can be shown (see [6]) that $\mathrm{d}^{*}$ is a vertex of the set $\mathcal{D}$ iff $\mathbf{d}^{*}$ is a dropping rate vector resulting from an Ordered HoL (O-HoL) priority service policy $\pi=\left(\pi_{1}, \pi_{2}, \ldots, \pi_{N}\right) ; \pi_{i} \in\{1,2, \ldots, N\}, \pi_{i} \neq \pi_{j}, 1 \leq i, j \leq N$. The index of $\pi_{i}$ indicates the order of the priority given to the $\pi_{i}$ source. None of the $\pi_{j}$ sources, $j>i$, may be served as long as packets from sources $\pi_{k}$, $k \leq i$, are present.

Fig. 1 provides a graphical illustration of the region $\mathcal{D}$ for the case of $N=2$ and $N=3$ sources. The extreme points correspond to QoS vectors $\mathrm{d}$ induced by the $N$ ! ordered HoL priority policies $\pi=\left(\pi_{1}, \pi_{2}, \ldots, \pi_{N}\right)$.

The region of achievable QoS is shaped by both the amount of available resources and the level of interference in the wireless channel. To illustrate this, consider the following example of two sources competing for $T$ slots in a TDMA frame. The source packet arrival processes are assumed to be mutually independent. Each arrival process is embedded at the frame boundaries. The number of packets generated (and requesting service) by a source in the current frame boundary is (probabilistically) determined by the present state of the underlying arrival process. The region of achievable QoS is only dependent on the state probability distribution, thus, correlation in the source packet ar- 


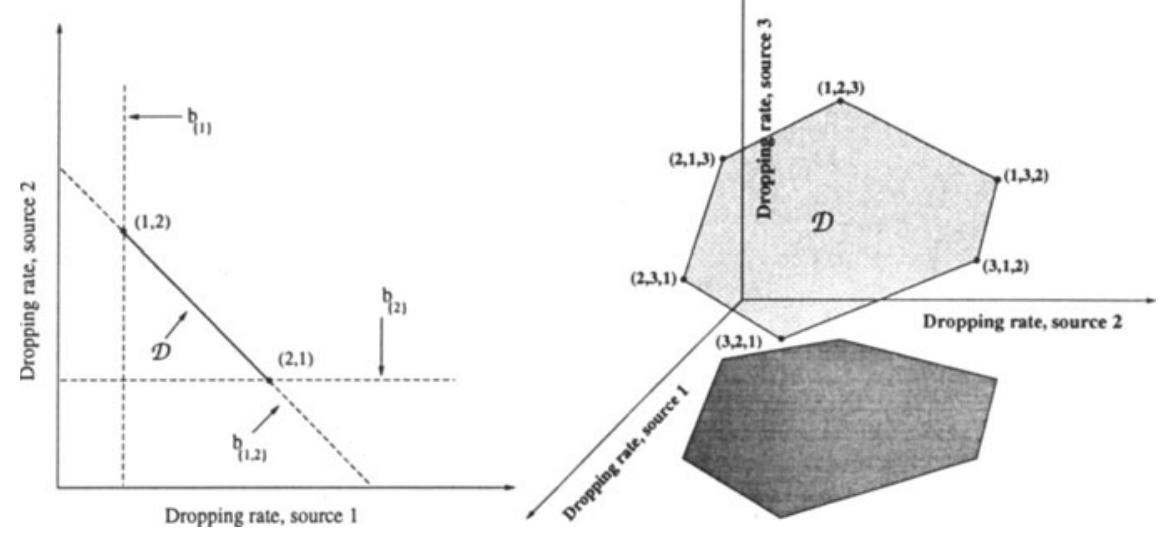

Figure 1 The region (polytope) $\mathcal{D}$ for a system with two and three sources under channel conditions $\beta=0.02$.

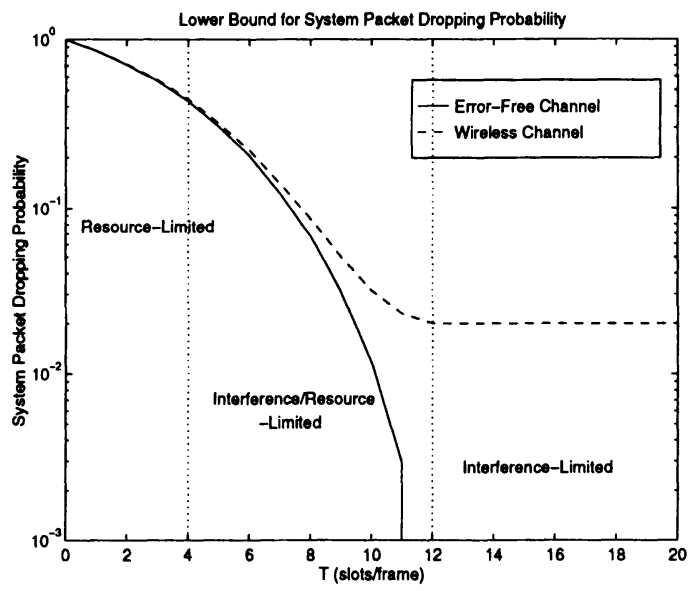

Figure 2 System packet dropping probability for in an error-free channel and a (non-ideal) wireless channel with channel conditions $\beta=0.02$.

rival process may be considered without affecting the region of achievable QoS or the analysis presented in this paper.

In this example, each VBR source is modeled by discrete-time batch Markov arrival process embedded at frame boundaries, with mean rate of 3.6 and 3.2 packets per frame, and variance of 2.04 and 3.36 packets per frame, respectively. In Fig. 2, the conserved system packet dropping probability $p_{S}=\frac{b_{S}}{E\left[\lambda_{S}(n)\right]}$ is plotted as a function of available resources $T$ (time slots) for an error-free channel $(\beta=0)$ and a wireless channel with channel quality, $\beta=0.02$.

As it can be seen in this figure, there are three distinct regions of operations: 
Resource-Limited, Interference/Resource-Limited and Interference-Limited. In the resource-limited region, the performance is primarily determined by the amount of available resources. This result is evident since the packet dropping probability for the system with the error-free channel and the (non-ideal) wireless channel are almost identical. In the interference-limited region, the dropping probability in the error-free channel is zero, while the performance in the wireless system is limited by the interference and given by $\beta=0.02$. The performance in the interference/resource-limited region is determined by both, the available resources and the level of interference in the channel. In this example, the system packet dropping probability in this region ranges from $10^{-1}-10^{-2}$, an operation region of interest for real-time applications. It is important to note (as shown earlier) that, in general, satisfying the system packet dropping rate (probability) is only necessary and not sufficient to guarantee that the target QoS vector is achievable*.

\subsection{Impact of Real-Time Data Link Control}

A Data Link Control Layer (DLC) can be added to combat the effects of the wireless channel in an interference/resource-limited or interference-limited system. Due to the real-time constraints of the supported applications; traditional automatic repeat request (ARQ) strategies are not possible. In this section, a real-time DLC protocol is proposed and the impact that this layer has on the region of achievable QoS is evaluated.

To combat the effects of interference, the real-time DLC protocol considered in this work will generate multiple copies of certain packets for transmission over the current frame. This strategy will improve the probability of correct reception (or reduce the probability of packet dropping at the receiver) while meeting the real-time service constraint. Copies are transmitted only during underloaded frames utilizing the remaining resources*. Transmitting a copy from a set $g$ during an overloaded frame would reduce the probability of packet dropping at the receiver for the set $g$, but would force an original packet from the complement set $\{S-g\}$ to be dropped at the source. As a result, the overall system dropping rate to increase. In view of the above discussion, if the objective of the real-time DLC protocol is to minimize the system packet dropping probability (or equivalently packet dropping rate), and therefore maximize system throughput, then multiple copies of packets can be sent only during underloaded frames - utilizing the remaining resources.

During underloaded frames, the number of copies generated by the the DLC is a function of the scheduling policy and the amount of remaining resources.

\footnotetext{
*In the special case of a homogeneous system, such as a cellular voice system, satisfying system performance is sufficient to guarantee that the target $\mathrm{QoS}$ vector is achievable. This result has been established in [6].

*The remaining resources in frame $n$ are defined to be $\left(T-\lambda_{S}(n)\right)$.
} 
A policy that attempts to "fairly" allocate the remaining resources among sources will result in the minimum system dropping rate. This result can be seen by letting $\mathbf{k}^{f}=\left(k_{1}^{f}, k_{2}^{f}, \ldots, k_{\lambda_{S}(n)}^{f}\right), 1 \leq k_{m}^{f} \leq T, 1 \leq m \leq \lambda_{S}(n)$, be a vector which determines the number of transmissions of each packet $m \in\left\{1,2, \ldots, \lambda_{S}(n)\right\}$. For a given $\lambda_{S}(n)$, the expected number of packets arriving at the receiver in error under this policy is equal to,

$$
\sum_{m=1}^{\lambda_{S}(n)} \beta_{m}^{k_{m}^{f}}
$$

where, $\sum_{m=1}^{\lambda_{S}(n)} k_{m}^{f}=T, 1 \leq k_{m}^{f} \leq T, \quad \forall m \in\left\{1,2, \ldots, \lambda_{S}(n)\right\}$. Since $\beta^{x}$ is a convex function of $x$, that is, $\gamma \beta^{a}+(1-\gamma) \beta^{b} \geq \beta^{\gamma a+(1-\gamma) b}$ for $0 \leq \gamma \leq 1$, then,

$$
\sum_{m=1}^{\lambda_{S}(n)} \beta^{k_{m}^{f}} \geq \lambda_{S}(n) \beta^{\frac{T}{\lambda_{S}^{(n)}}}
$$

Since (9) is minimum when $k_{m}^{f}=\frac{T}{\lambda_{S}(n)}=k$, (9) is minimized when each sources $i$ receives $\lambda_{i}(n) k=\frac{\lambda_{i}(n)}{\lambda_{S}(n)} T$ slots for transmission of the $\lambda_{i}(n)$ packets and its copies. In this sense the policy is "fair".

However, due to the granularity in the system (that is, resources can be allocated only in integer multiples), the following is considered. During underloaded frames, let each packet be transmitted $R \triangleq\left\lfloor\frac{T}{\lambda_{S}(n)}\right\rfloor, R \geq 1$, number of times, where [.] denotes the integer part, and let $X \triangleq\left(T-\lambda_{S}(n) R\right)$ be the number of packets that can be transmitted one additional time, $(R+1)$. The system dropping rate is then conserved regardless of which set of sources the packets that are transmitted one additional time belong to. Allocating the additional retransmission during underloaded frames according to a policy allows for further diversification of the resulting QoS vector delivered, while still satisfying the requirement of minimum system dropping rate. Therefore, under any fair-work-conserving policy, the system dropping rate (given below) is minimum and also conserved.

The results from Section 3.1 can be modified to account for the impact of the DLC protocol. Considering the effects of the physical channel, the scheduling policy and the real-time DLC protocol, the number of packets from source $i$ dropped in frame $n$ is given by,

$$
d_{i}^{f}(n)=\left\{\begin{array}{ll}
\sum_{m=1}^{\lambda_{i}(n)} \prod_{q=1}^{R+1_{m}^{f}} Z_{m}^{q} & \text { if } \lambda_{S}(n) \leq T \\
\lambda_{i}(n)-a_{i}^{f}(n)+\sum_{m=1}^{a_{i}^{f}(n)} Z_{m} & \text { if } \lambda_{S}(n)>T
\end{array}, 0 \leq i \leq N\right.
$$


$1_{m}^{f} \in\{0,1\}$ and indicates the dependency of the additional transmission of a copy of packet $m$ on the fair policy $f$, where $\sum_{m=1}^{\lambda_{S}(n)} 1_{m}^{f}=X, \forall f . Z_{m}^{q}$ is an indicator function associated with the $q^{\text {th }}$ transmission of the $m^{\text {th }}$ packet from source $i$. In this case, $E\left[d_{i}^{f}(n)\right]=d_{i}^{f}$ (mean per frame packet dropping rate) is the QoS provided to the network layer by the underlying DLC, MAC and physical channel.

The region of achievable QoS vectors when the above DLC layer is present can be derived by modifying the lower bounds given by (4) and (5), to account for the impact of the real-time DLC protocol. As previously stated the system dropping rate under all fair-work-conserving polices $f$ is conserved, it is given by,

$$
\begin{array}{r}
b_{S}=\left\{E\left[\lambda_{S}(n) \mid \lambda_{S}(n)>T\right]-T(1-\beta)\right\} P\left(\lambda_{S}(n)>T\right) \\
+E\left[X \beta^{R+1} \mid \lambda_{S}(n) \leq T\right] P\left(\lambda_{S}(n) \leq T\right) \\
+E\left[\left(\lambda_{S}(n)-X\right) \beta^{R} \mid \lambda_{S}(n) \leq T\right] P\left(\lambda_{S}(n) \leq T\right) .
\end{array}
$$

With the addition of this DLC protocol, the lower bound $b_{g}$ for the aggregate packet dropping rate for sources in $g$ under any fair-work-conserving policy $f$ is determined to be,

$$
\begin{aligned}
b_{g}= & \left\{E\left[\lambda_{g}(n) \mid \lambda_{g}(n)>T\right]-T(1-\beta)\right\} P\left(\lambda_{g}(n)>T\right) \\
& +E\left[\min \left[\lambda_{g}(n), X\right] \beta^{R+1} \mid \lambda_{g}(n) \leq T\right] P\left(\lambda_{g}(n) \leq T\right) \\
+ & E\left[\max \left[0, \lambda_{g}(n)-X\right] \beta^{R} \mid \lambda_{g}(n) \leq T\right] P\left(\lambda_{g}(n) \leq T\right) .
\end{aligned}
$$

The expected value in (12) is with respect to $\left\{\lambda_{g}(n), \lambda_{S}(n)\right\}$, which is easily computed since $P\left(\lambda_{g}(n)=i, \lambda_{S}(n)=j\right)=P\left(\lambda_{g}(n)=i\right) P\left(\lambda_{\{S-g\}}(n)=j-i\right)$. The expressions in (11) and (12) reduce to (4) and (5) respectively, for $R$ fixed and equal to 0 . The extreme points in this case correspond to FairOrdered-HoL (F-O-HoL) priority service policies. A F-O-HoL service policy is an O-HoL service policy $\pi=\left(\pi_{1}, \pi_{2}, \ldots, \pi_{N}\right)$ in which the additional retransmissions are also allocated according to $\pi$.

With the addition of the real-time DLC protocol, the region of achievable QoS for an interference/resources or interference-limited system can be improved compared to the system not employing the DLC protocol. The impact the real-time DLC protocol has on the system packet dropping probability for the example described in Section 3.1 is shown in Fig. 3.

In this figure, the performance of the two systems is compared to that in an error-free environment. As it can be seen in this figure, the system employing the real-time DLC protocol induces a lower packet dropping probability than the system without. The impact is most significant in the interference/resource- 


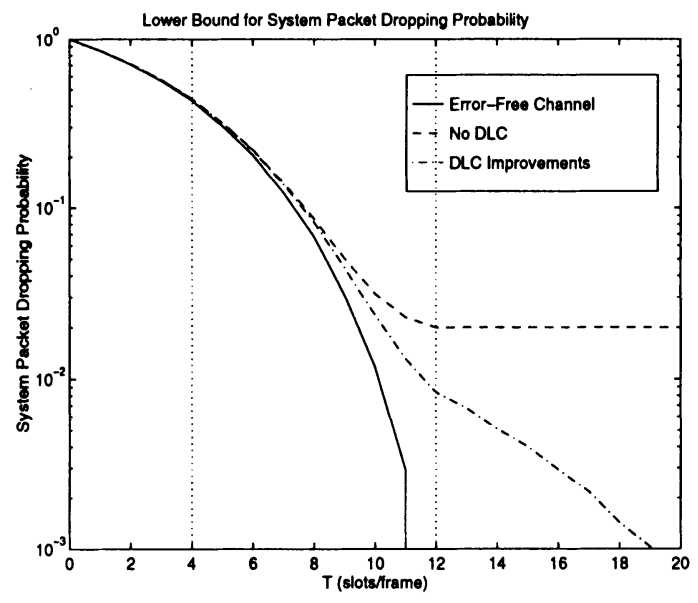

Figure 3 The impact of the real-time DLC protocol on the system packet dropping probability in a wireless channel with channel conditions $\beta=0.02$.

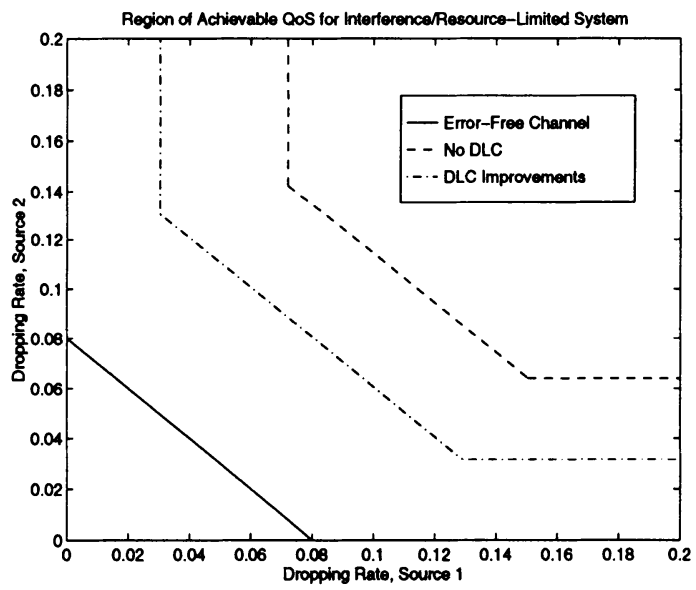

Figure 4 The impact of the real-time DLC protocol on the system packet dropping probability in a wireless channel with channel conditions $\beta=0.02$.

limited and interference-limited regions. In these regions the system with the real-time DLC takes advantage of the remaining resources and can reduce the packet dropping probability.

The impact that the real-time DLC has on the region of achievable QoS $\mathcal{D}$ is illustrated in Fig. 4. In this figure, $\mathcal{D}$ is derived for the system of sources given in Section 3.1 and with $T=10$ slots. Thus, according to Fig. 2, the system is interference/resource-limited. As it can be seen, a larger collection of QoS vectors can be accommodated. 


\section{A CLASS OF POLICIES DELIVERING ANY ACHIEVABLE QOS VECTOR IN $\mathcal{D}$}

In this work, the region of achievable $\mathrm{QoS} \mathcal{D}$ leads to a class of scheduling polices capable of delivering any achievable performance. The result follows from that fact that $\mathcal{D}$ can be written as a convex combination of the extreme points (vertices) $\mathrm{d}_{e x t-i}$ of $\mathcal{D}$. That is if $\mathbf{d} \in \mathcal{D}$, then $\mathbf{d}=\sum_{i=1}^{N !} \alpha_{i} \mathbf{d}_{e x t-i}$ for some $\boldsymbol{\alpha}=\left(\alpha_{1}, \alpha_{2}, \ldots, \alpha_{N !}\right)$ where $\alpha_{i} \geq 0,1 \leq i \leq N !, \sum_{i=1}^{N !} \alpha_{i}=1$. Therefore, by selecting the F-O-HoL priority policy that induces the extreme point $\mathbf{d}_{e x t-i}$ of $\mathcal{D}$ with probability $\alpha_{i}$, any QoS vector in $\mathcal{D}$ can be delivered. This class of policies is referred to as a mixing F-O-HoL priority policy. Typically, several mixing priority polices exist that can deliver the target dropping rate vector. This allows for the incorporation of additional constraints representing other desirable qualities of the policies. Functions of interest may be minimized subject to the constraints presented to guarantee the delivery of the target QoS vector. For instance, among all the mixing policies inducing $d$, the one which minimizes the variance of the service provided to certain sources may be identified.

\section{CONCLUSION}

In this work* ${ }^{*}$ the region of achievable QoS has been precisely described for a system of heterogeneous real-time VBR sources competing for an unreliable wireless channel. The QoS has been defined in terms of a packet dropping probability (or equivalently packet dropping rate). Packets from sources in the system were dropped as a result of delay violations and channel induced errors. As a consequence, it has been shown that the region of achievable QoS is shaped by both the interference in the physical channel and the amount of available resources. In addition, a simple DLC protocol has been proposed to combat the effects of the wireless channel while still satisfying the realtime service constraints of the associated applications. The results presented in this paper illustrate the positive impact of the employed DLC protocol on the region of achievable QoS.

\section{REFERENCES}

[1] N.D. Newman, R. Ganesh, K. Joseph, and D. Raychaudhuri. Packet CDMA Versus Dynamic TDMA for Multiple Access in an Integrated Voice/Data PCN. IEEE Journal on Selected Areas in Communications, 11(6):870-884, August 1993.

*Research supported in part by the National Science Foundation under Grant NCR 9628116. 
[2] D. Raychaudhuri and N. Wilson. ATM-Based Transport Architecture for Multiservices Wireless Personal Communication Networks. IEEE Journal of Selected Areas Communications, 12(8):1401-1414, October 1994.

[3] B. Walke, D. Petras, et al. Wireless ATM: Air Interface and Network Protocols of the Mobile Broadband System. to appear in IEEE Personal Communication Magazine.

[4] G. Anastasi, D. Grillo, and L. Lenzini. An Access Protocol for Speech/Data/Video Integration in TDMA-Based Advanced Mobile Systems. IEEE Journal of Selected Areas in Communications, 15(1), January 1997.

[5] C. Chang, K. Chen, M. You, and J. Chang. Guaranteed Quality-of-Service Wireless Access to ATM Networks. IEEE Journal of Selected Areas in Communications, 15(1), January 1997.

[6] J. Capone and I. Stavrakakis. Achievable QoS and scheduling Policies in Integrated Services Wireless Networks. Performance Evaluation, 26 and 27(1), October 1996.

[7] J. Capone and I. Stavrakakis. Delivering Diverse Delay/Dropping QoS Requirements in a TDMA Environment. In Proceedings of ACM MobiCom, Budapest, Hungary, Sept. 1997.

[8] J. Bibb Cain and D. McGregor. A Recommended Error Control Architecture for ATM Networks with Wireless Links. IEEE Journal of Selected Areas in Communications, 15(1), January 1997.

[9] J. Capone and I. Stavrakakis. Achievable QoS in an Interference/Resource-Limited Shared Wireless Channel. submitted to IEEE Journal of Selected Areas on Communications.

\section{BIOGRAPHY}

Jeffrey M. Capone received the B.S.E.E. degree from the University of Vermont, Burlington, VT, in 1992, the M.S.E.E. and Ph.D. degree from Northeastern University, Boston, MA, in 1995 and 1997, respectively. In 1997, he joined the faculty of Electrical Engineering at Arizona State University. His primary research interest is in the design and analysis of controlling policies for bandwidth management in wireless communication networks.

Ioannis Stavrakakis received the Diploma in Electrical Engineering from the Aristotelian University of Thessaloniki, Thessaloniki, Greece, 1983, and the Ph.D. degree in Electrical Engineering from the University of Virginia, 1988. In 1988, he joined the faculty of Computer Science and Electrical Engineering at the University of Vermont as an assistant and then associate professor. Since 1994, he has been an associate professor of Electrical and Computer Engineering at Northeastern University, Boston. His research interests are in stochastic system modeling, teletraffic analysis and discrete-time queueing theory. 